\title{
Incorporación de un Proyecto de Aprendizaje-Servicio para Estudiantes de Grado Aplicado a la Gestión de la Calidad Medioambiental
}

\section{Daniel Ferrández ${ }^{a}$, Irantzu Recalde ${ }^{b}$ y Engerst Yedra ${ }^{c}$}

${ }^{a}$ Departamento de Ingeniería de Organización, Administración de Empresas y Estadística, Universidad Politécnica de

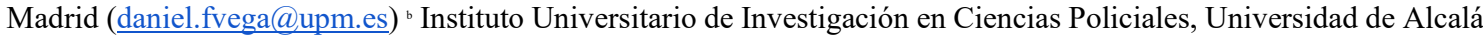
de Henares (iratzu.recalde@uah.es) •Departamento de Tecnología de la Edificación, Universidad Politécnica de Madrid (e.yedra@alumnos.upm.es)

\begin{abstract}
Through Service-Learning (SL), the realization of useful service for the community, and the acquisition of content and curricular skills combined actively. This work proposes the design of an $S L$ project for its application with undergraduate students in engineering. It has addressed explicitly from the subject of Environmental Quality Management, the planning, and implementation by students of a service that contributes to the advancement of any of the Sustainable Development Goals proposed by the United Nations. What is presented in this research is the achievements obtained in the process of implementing this methodology, as well as its organization by teachers and how their activities were adapted to work according to this pedagogical initiative. It has verified that the involvement of students in the social part of the methodology is of vital importance since what is promote goes beyond the curricular content of a degree subject.
\end{abstract}

Keywords: Service Learning, Sustainable Development Goals, environmental quality, competencies, educational project.

\section{Resumen}

A través del Aprendizaje-Servicio (ApS) se combina de forma activa la realización de un servicio de utilidad para la comunidad y la adquisición de contenidos y competencias curriculares. En este trabajo se propone el diseño de un proyecto de ApS para su aplicación con estudiantes de grado en ingeniería. Más concretamente, se aborda desde el tema de Gestión de la Calidad Medioambiental, la planificación y puesta en práctica por parte del alumnado de un servicio que contribuya al avance en alguno de los Objetivos para el Desarrollo Sostenible propuestos por las Naciones Unidas. Dado que es un proyecto en curso, lo que se presenta en esta investigación son los logros obtenidos en el proceso de puesta en marcha de esta metodología, asi como, su organización por parte de los docentes y la manera en que se han adaptado su actividades para trabajar conforme a esta iniciativa pedagógica. Se ha podido corroborar la importancia de la implicación del alumnado en la parte social de la metodología, pues lo que se promueve va más allá de los contenidos curriculares de una asignatura de grado.

Palabras clave: Aprendizaje por Servicio, Objetivos de Desarrollo Sostenible, calidad medioambiental, competencias, proyecto educativo.

\section{Introducción}

El contexto de esta investigación surge tras la creación de la oficina de Aprendizaje Servicio (ApS) de la Universidad Politécnica de Madrid (UPM) en enero del año 2020. Dicha oficina lanzó la convocatoria 
Proyectos de Aprendizaje Servicio para el presente curso académico (2019-2020), y por lo tanto, se quiso participar en ella, adaptando parte de los contenidos curriculares de la asignatura de Gestión y Control de la Calidad del doble grado de Ingeniería Informática y Administración de Empresas de la UPM.

El motivo de la elección de la asignatura de Gestión y Control de la Calidad es debido a la gran diversidad de bloques temáticos que ofrece relacionados con los 17 Objetivos para el Desarrollo Sostenible (ODS) impulsados por las Naciones Unidas. Esta amplia oferta posibilita la inclusión de varios de estos objetivos para dar continuidad a la agenda de desarrollo, adaptándose a los distintos intereses personales de los educandos, y permitiendo así que el alumnado se involucre en el proceso búsqueda de iniciativas transformadoras para el desarrollo sostenible.

Así pues, conscientes del potencial del alumnado del Doble Grado de Ingeniería Informática y Administración de Empresas, se ha decidido llevar a cabo una experiencia educativa con aplicación directa a la universidad, de forma práctica y colaborativa con la institución. En la Tabla 1, se realiza una síntesis que permite encuadrar el marco de actuación del presente trabajo.

Tabla 1. Información detallada del contexto de la propuesta.

\begin{tabular}{ll}
\hline Universidad & Universidad Politécnica de Madrid (UPM) \\
Centro & Escuela Técnica Superior de Ingeniería Informática \\
Titulación & Doble Grado de Ingeniería Informática y ADE \\
Asignatura / Curso & Gestión y Control de la Calidad / $3^{\circ}$ Curso (6 ${ }^{\circ}$ Semestre) \\
Tema tratado & Gestión de la Calidad Medioambiental y ODS. \\
Número de estudiantes & 25 \\
\hline
\end{tabular}

Conviene indicar que la aplicación de esta metodología docente guarda relación con uno de los objetivos principales de la Gestión de la Calidad Total, como es el caso de la mejora continua que pretende mediante cambios graduales y frecuentes mejorar las acciones y las cosas dentro de la empresa (Fernández, 2008). En este sentido, el estudiantado al verse implicado con la universidad (más concretamente con aplicación en el Campus de Montegancedo de la UPM), aumenta su motivación y aplica los contenidos de la asignatura de forma directa, poniendo en práctica todo los conceptos aprendidos a través de un proceso de mejora social en su entorno. Se trata por tanto de hacer más dinámica la asignatura, aumentando la participación y capacidad de comunicación del alumnado, así como, fomentar el pensamiento crítico y su concienciación con la sociedad a través del trabajo en equipo.

El ApS es una propuesta educativa, metodológica y pedagógica en la que se practica de manera combinada el aprendizaje y el servicio, bajo el respaldo de un proyecto (De Santiago et al., 2019). Dicho aprendizaje es un aprendizaje con significado, porque precisamente mediante el servicio, se relaciona lo aprendido con el contexto físico-social en el que el estudiante lo aplica, favoreciendo un aprendizaje activo y en profundidad (Maroto, 2018; López-Doria y Martín, 2018). De hecho, una de sus bondades es la posibilidad de transmitir la importancia y responsabilidad del autoaprendizaje para la correcta realización del servicio, además del crecimiento en valores que obtiene tanto el alumnado como el profesorado, al verse estos últimos también implicados en los proyectos y servicios que desarrollan los y las estudiantes (Del Río, 2016). Tal y como defiende Puig (2018), es una propuesta muy flexible, lo que favorece su aplicación en múltiples situaciones y realidades. Un aspecto que se considera fundamental es la compenetración de esta propuesta educativa con la ética de los cuidados, tan necesaria para la aplicación del servicio, ya que el cuidado de las personas y el entorno es, en definitiva, el cuidado de la vida (Uruñuela, 2018). Una manera de resumir gráficamente esta definición se presenta en la Figura 1, por otro lado, Uruñuela (2018: 22) expone la metodología con el siguiente ejemplo: 
Todos/as hemos visto imágenes de incendios en verano, imágenes espectaculares de cómo se quemaba el monte. El paisaje que queda tras el incendio es desolador: todo quemado, todo destruido, un desastre. Y, pasado un tiempo, es frecuente ver a chicos y chicas y jóvenes efectuando tareas de recuperación y reforestación de la zona. Si analizamos lo que hacen estos alumnos/as, vemos que plantar árboles donde se necesita es un acto solidario. A su vez, estudiar las características del bosque mediterráneo, analizar su degradación y su repercusión en el incremento de los incendios es una actividad de aprendizaje. Comprometerse en una campaña de reforestación, aplicando lo estudiado en las distintas materias relacionadas con el medio ambiente, es poner en marcha un proyecto de ApS.

De acuerdo con Puig (2018), el aprendizaje-servicio cumple con creces criterios de calidad metodológica y formal, por lo que es una oportunidad para la comunidad educativa extraer las fortalezas y oportunidades que brinda dicha metodología en lo referido a la experiencia formativa, sin duda rica y compleja, vinculada a la realidad. Es altamente recomendable para la conexión de jóvenes con otros colectivos y tejidos sociales, lo que permite encuentros cara a cara que potencian las relaciones sociales, el redescubrirse y desestigmatizarse, así como una mayor inclusión en la sociedad en la que se trabaja (López-Doria y Martín, 2018). El ApS potencia y fomenta el sentimiento de pertenencia tanto a la institución de la que nace el aprendizaje, como aquella organización, sector o colectivo en el que se presta el servicio. Los jóvenes viven en primera persona la utilidad de su aprendizaje. Viven como ellos son motor del cambio, motor de mejora y motor de aprendizaje para otras personas, experiencias con las que la mayor parte de ellos no se encuentran familiarizados.

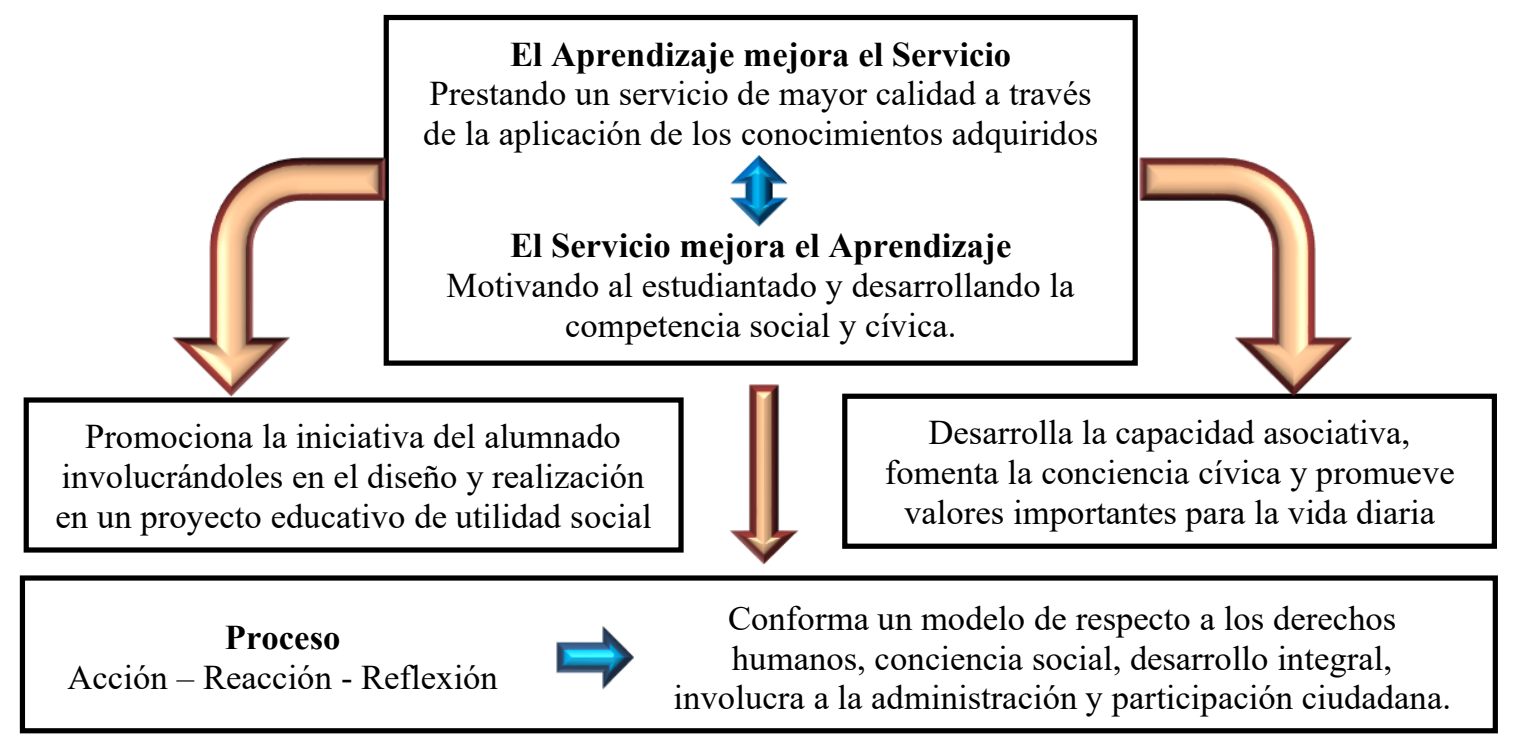

Fig.1. Beneficios del Aprendizaje por Servicio. Fuente: Elaboración propia a partir de Medina (2012).

Por último, resaltar que, entre los beneficios que aporta esta metodología al centro educativo en el que se implanta, Uruñuela (2018) destaca que, en primer lugar, pone en primer plano la finalidad "original" de la educación, tantas veces olvidada entre evaluaciones curriculares y calificaciones: formar personas solidarias, respetuosas y tolerantes, algo perfectamente compatible con la excelencia académica. En segundo lugar el citado autor señala que, mediante esta metodología, se cubre el trabajo de las competencias sociales y ciudadanas, también recogidas en el plan del Espacio Europeo de Educación Superior (EEES) y, en tercer y último lugar, esta metodología revierte en una convivencia positiva en el centro educativo, al fomentar las buenas relaciones con uno mismo, con otras personas y con el entorno. 


\section{Objetivos}

El objetivo general de este trabajo es acercar a los profesionales de la educación una propuesta metodológica para abordar la planificación de un trabajo para asignaturas de grado en enseñanzas técnicas, incorporando la metodología ApS y abordando distintos Objetivos de Desarrollo Sostenible de los propuestos por las Naciones Unidas. Para ello, se describe la experiencia llevada a cabo con el alumnado de tercer curso del Doble Grado en Ingeniería Informática y ADE de la UPM, y cuya finalidad no es otra que aumentar la motivación y mejorar el proceso de aprendizaje del estudiantado.

Por otra parte, de manera más específica se pretende:

- Desarrollar la competencia social y cívica del estudiantado que cursa estudios de grado en ingeniería, promoviendo valores importantes para la mejora de la ciudadanía a través de la contribución en el desarrollo de alguno de los ODS propuestos para el año 2030.

- Reforzar el sentimiento de identidad del estudiantado en torno a su afiliación con la Universidad, tomando conciencia de las acciones que se pueden llevar a cabo para mejorar la calidad de la institución y ayudando mediante su puesta en servicio a concienciar a gran parte de la comunidad educativa de la UPM.

- Favorecer que los estudiantes entren en contacto con la realidad de su entorno y apliquen directamente los conocimientos adquiridos en la asignatura al servicio de la comunidad educativa.

\section{Desarrollo de la innovación}

\section{SEIS PASOS a seguir para la planificación de un Proyecto ApS}

Conviene $\frac{\text { INICIO }}{\text { adaptar }}$ algún un
proyecto ya comenzado y del
que se tenga información.
Es importante encontrar las
necesidades, de forma clara y
concisa. Para poder enfocar el
servicio de manera que se dé
respuesta a una necesidad real.

1

\section{PLAN - ACCIÓN}

Concretar con todo detalle las acciones, responsabilidades, recursos, evaluación y el seguimiento. Adaptándose a las necesidades.

La metodología del ApS implica un empoderamiento de los participantes.

\section{COLABORACIÓN}

Es necesario contar con la colaboración de entidades que estén implicadas con la necesidad detectada en la fase inicial.

Es conveniente a su vez poner por escrito los acuerdos logrados con la institución.

2

\section{ANÁLISIS}

Programar una reflexión sobre las acciones realizadas para asentar el aprendizaje.

Reconocer el esfuerzo de todos los participantes del proyecto $\mathrm{y}$ en especial del alumnado, celebrando los resultados positivos.
SERVICIO A REALIZAR

Describiendo aquello que vamos a hacer, las respuestas y sus características.

Conocer de antemano los conocimientos requeridos, y que tipo de aprendizajes y que competencias se van a trabajar con el proyecto.

\section{3}

\section{MEJORA}

Incluir aquellos cambios en el proyecto que se consideren necesarios y que se hayan venido reflejando durante su ejecución.

Incorporar el proyecto ApS en la guía docente $y$ darle continuidad en el tiempo.

Fig.2. Seis pasos para la realización de un proyecto ApS. Fuente: elaboración propia a partir de Puig (2009) (cc) EY-NC-ND 2020, Universitat Politècnica de València 
A diario escuchamos noticias reflejando el deterioro del planeta ocasionado por la acción humana. Es importante la concienciación ciudadana desde todos los niveles educativos en materia de sensibilización medioambiental, sensibilizándonos con la necesidad de respetar y colaborar para la consecución de los Objetivos de Desarrollo Sostenible que nos marcan las Naciones Unidas. De esta forma, se necesita llevar a cabo una planificación detallada sobre la acción-servicio a realizar, para ello se proponen los seis pasos reflejados en la Figura 2.

Así pues, y en concordancia con los pasos descritos en la Figura 2, se estructuraron de manera lógica los siguientes pasos para la organización del proyecto de Aprendizaje-Servicio en la asignatura de Gestión y Control de la Calidad:

1. Tradicionalmente se venía realizando un trabajo en la asignatura con ponderación del $10-20 \%$ que consistía en la realización de un Plan de Calidad. Los resultados y motivación del estudiantado hacia el mismo eran escasos y no abarcaba la totalidad de las competencias a tratar. Por otra parte, las sanciones impuestas por la UE a España en materia de gestión energética y de residuos en los últimos años habían servido como motivación de diferentes Trabajos Fín de Grado en la UPM. Es por este motivo, que se ha decidido implantar un proyecto de Aprendizaje-Servicio desde la asignatura de Gestión y Control de la Calidad para alumnos del Doble Grado de Ingeniería informática y $\mathrm{ADE}$, que ayude a involucrar a los profesionales técnicos en los problemas de sostenibilidad que afronta el planeta, acercando los contenidos de la asignatura a esta necesidad y que culminan con la contribución a una serie de Objetivos para el Desarrollo Sostenible fijados por las Naciones Unidas.

2. Desde el primer momento se contó con el apoyo del profesorado del $6^{\circ}$ Semestre del Doble Grado para la puesta en marcha del proyecto, así como con la colaboración del personal del Campus. Además, se llevó a cabo una serie de explicaciones en el aula acerca de qué es la metodología ApS para mejorar la implicación del alumnado con el proyecto, durante esta fase se contó con el apoyo de personal investigador experto en el área proveniente de la Universidad de Alcalá, quien compartió diferentes experiencias realizadas con anterioridad en su centro y se ofrecieron ejemplos de actuación que pudieran inspirar a los alumnos y alumnas.

3. A continuación, se fijaron líneas de actuación para que el estudiantado desarrollase su trabajo en torno a uno de los siguientes ODS planteados:

- $\quad$ Educación de Calidad (Objetivo 4)

- Agua Limpia y Saneamiento (Objetivo 6)

- Energía Asequible y No Contaminante (Objetivo 7)

- Producción y Consumo Responsables (Objetivo 12)

- Acción por el Clima (Objetivo 13)

- Vida Submarina (Objetivo 14)

- Vida de Ecosistemas Terrestres (Objetivo 15)

La elección de estos Objetivos y no otros es su proximidad con el temario relacionado con la Gestión de la Calidad Medioambiental impartido en la asignatura. En cuanto a este apartado, el profesorado tuvo que reestructurar la distribución temática de la asignatura ya que era necesario que el estudiantado adquiriese los siguientes conocimientos previos:

- Herramientas para la gestión de la calidad, y en particular el concepto de Círculo de Calidad como metodología de trabajo en equipo y que puede describirse de la siguiente manera: "[...] un pequeño grupo de personas que trabajan en tareas similares dentro de una estructura formal de la empresa, dotado de un alto grado de autonomía, que persigue, de forma sistemática y continua, mejoras incrementales en el trabajo en

(c)) BY-NC-ND 2020, Universitat Politècnica de València

Congreso In-Red (2020) 
aspectos relacionados con la calidad, la seguridad, la productividad, el medio ambiente o las condiciones de trabajo" (Miranda, Chamorro y Rubio, 2017: 114).

- Filosofía de la Mejora Contínua como modo de operar durante el trabajo y que se de define como: "Un Proceso de carácter dinámico que implica la realización de cambios graduales, pero muy frecuentes, estandarizando los resultados obtenidos tras cada mejora alcanzada. Su idea básica es que siempre es posible hacer mejor las cosas [...]" (Miranda, Chamorro y Rubio, 2017: 77).

Así pues, se pretende que el estudiantado adquiera con la realización de esta práctica metodológica las siguientes competencias en la asignatura:

- Aplicar técnicas y procedimientos de gestión, control y aseguramiento de la calidad.

- Que los estudiantes sean capaces de trabajar en el ámbito de la administración y dirección de empresas aplicando criterios de calidad y sostenibilidad.

4. Llegado este punto, se procedió a la formación de grupos de trabajo (círculos de calidad) y a la discusión de las propuestas de acción elegidas por el alumnado. Entre otras propuestas de actuación en el Campus se recogieron: gestión del transporte universitario y acciones para reducir la emisión de contaminantes, mapeado del Campus buscando puntos de reciclaje y elaboración de propuestas de mejora en organización de lugares de recogida de residuos, análisis del consumo energético y planificación hacia una transición energética sostenible, etc. En el momento de aprobación de la propuesta, previa discusión del borrador que se envió con anterioridad al docente, los estudiantes repartieron sus responsabilidades y se adaptó el proyecto en base al alcance previsto de la acción a desarrollar. En todo momento se potenció el protagonismo del alumnado y su autodeterminación en la toma de decisiones, valorando sus propuestas y erigiéndoles como artífices de su trabajo.

5. Una vez realizadas las acciones o propuestas de acción los distintos grupos de estudiantes formados entregaron un informe, donde se evaluó positivamente: los conocimientos aplicados que guardaban relación con la asignatura, el compromiso en la contribución al ODS elegido, los materiales y recursos utilizados, así como la forma de llevar a cabo las acciones elegidas. En este informe se incluyeron también unas conclusiones sobre lo aprendido mediante la puesta en práctica de la metodología ApS.

Además, para el caso concreto del presente curso académico y con motivo de los inconvenientes ocasionados por el COVID-19, se reconoció y premió de manera especial el esfuerzo y originalidad, así como, la capacidad de trabajo y superación que el estudiantado ha mostrado para culminar con éxito la propuesta.

6. Finalmente, se propuso la realización de un cuestionario obligatorio a través de la plataforma Moodle, en el que se recogieran las impresiones y propuestas de mejora tras su experiencia con el trabajo de la asignatura y la metodología ApS. En base a estos resultados, se plantearán cambios en la organización y gestión del trabajo para futuros cursos académicos, incluyéndose aquellas observaciones y pautas que se consideren constructivas.

\section{Resultados}

La planificación de la asignatura se realizó de forma coherente con los objetivos planificados y en base al aprendizaje que se quería lograr con los estudiantes a través de la aplicación de la metodología ApS. De esta manera, en la temporalización de las actividades recogida en la Tabla 2 se ha tenido en cuenta la organización de las sesiones teóricas, así como las preguntas fundamentales de para qué se hace esa actividad y cómo se puede lograr, en base siempre a la metodología ideada. 
Tabla 2. Temporalización para la entrega y realización de actividades ligadas al proyecto ApS.

\begin{tabular}{|c|c|c|c|c|}
\hline Actividad & Febrero & Marzo & Abril & Mayo \\
\hline Aproximación al ApS & $18 / 02 / 20$ & & & \\
\hline Formación de equipo de trabajo & $25 / 02 / 20$ & & & \\
\hline Envío de la propuesta de servicio unidad a un ODS & & $24 / 03 / 20$ & & \\
\hline Devolución de la propuesta corregida & & $31 / 03 / 20$ & & \\
\hline $\begin{array}{l}\text { Envío de un borrador del informe sobre la marcha y } \\
\text { aplicación de la propuesta anterior. }\end{array}$ & & & $14 / 04 / 20$ & \\
\hline Devolución del borrador corregido. & & & $21 / 04 / 20$ & \\
\hline Entrega del informe final. & & & & \\
\hline Evaluación de la experiencia a través del cuestionario & & & & 2 \\
\hline
\end{tabular}

Con esta idea, se quiere transmitir la importancia de una buena planificación inicial del semestre en base a la propuesta, ya que si se analiza previamente cuál será la organización de las clases, los resultados son más previsibles y es más probable la obtención de una evaluación satisfactoria. En este sentido, la aplicación de la metodología ApS en la asignatura en cuestión, está ligada a la contribución de la mejora del entorno y garantizar una mayor conformidad e índice calidad en el Campus de la universidad a través de la involucración del alumnado en el desarrollo de alguno de los ODS propuestos de inicio. Se consigue así una educación en valores, que sólo pueden ser aprendidos y asimilados desde la práctica.

Para la consecución del éxito de acuerdo con lo planificado el estudiantado debe conocer de antemano cómo va a ser evaluado, y conviene por tanto, ofrecer una rúbrica de evaluación donde se reflejen aquellos aspectos que serán tenidos en cuenta para la obtención de una calificación numérica final. Para el caso concreto de este trabajo se realizó la siguiente rúbrica de evaluación mostrada en la Tabla 3.

Tabla 3. Rúbrica de evaluación orientativa para el proyecto ApS de Gestión de la Calidad Medioambiental y ODS.

\begin{tabular}{|c|c|c|c|c|c|}
\hline ÍTEM & (4) Excelente & (3) Bien & (2) Aceptable & (1) Deficiente & Total \\
\hline $\begin{array}{l}\text { Participación / } \\
\text { reparto de tareas }\end{array}$ & $\begin{array}{l}\text { Todas participan } \\
\text { con entusiasmo }\end{array}$ & $\begin{array}{l}3 / 4 \text { partes } \\
\text { participan con } \\
\text { entusiasmo }\end{array}$ & $\begin{array}{l}1 / 2 \text { participa } \\
\text { activamente }\end{array}$ & $\begin{array}{c}\text { Solo una o } \\
\text { ninguna persona } \\
\text { participa } \\
\text { activamente }\end{array}$ & \\
\hline Responsabilidad & $\begin{array}{l}\text { Todas comparten } \\
\text { por igual la } \\
\text { responsabilidad } \\
\text { sobre la tarea }\end{array}$ & $\begin{array}{l}\text { 3/4 del grupo } \\
\text { comparten } \\
\text { responsabilidad }\end{array}$ & $\begin{array}{l}1 / 2 \text { comparte } \\
\text { responsabilidad }\end{array}$ & $\begin{array}{l}\text { La } \\
\text { responsabilidad } \\
\text { recae sobre una o } \\
\text { ninguna persona }\end{array}$ & \\
\hline $\begin{array}{l}\text { Originalidad / } \\
\text { Creatividad }\end{array}$ & $\begin{array}{c}\text { Trabajo muy } \\
\text { creativo y original }\end{array}$ & $\begin{array}{c}\text { Trabajo creativo } \\
\text { y original }\end{array}$ & $\begin{array}{c}\text { Trabajo poco } \\
\text { creativo/original }\end{array}$ & $\begin{array}{l}\text { Nada creativo ni } \\
\text { original }\end{array}$ & \\
\hline $\begin{array}{l}\text { Alcance / } \\
\text { Repercusión del } \\
\text { servicio/acciones } \\
\text { realizadas }\end{array}$ & $\begin{array}{l}\text { Alcance muy } \\
\text { elevado, gran } \\
\text { difusión }\end{array}$ & Alcance elevado & Poco alcance & Nada de alcance & \\
\hline $\begin{array}{l}\text { Aplicación de } \\
\text { conocimientos } \\
\text { adquiridos en } \\
\text { asignatura }\end{array}$ & $\begin{array}{l}\text { Se ha aplicado de } \\
\text { manera excelente }\end{array}$ & $\begin{array}{l}\text { Se aplica bien } \\
\text { conocimiento } \\
\text { extraído de la } \\
\text { asignatura }\end{array}$ & $\begin{array}{l}\text { Aplicación } \\
\text { regular de } \\
\text { conocimiento }\end{array}$ & $\begin{array}{l}\text { No hay / casi no } \\
\text { hay aplicación de } \\
\text { conocimiento }\end{array}$ & \\
\hline
\end{tabular}


No obstante, se debe indicar que la rúbrica anterior tiene un carácter orientativo, pero no limitativo. Es decir, que se puede ampliar y adaptar a las necesidades del profesorado en base al proyecto a realizar. El empleo de esta herramienta flexibiliza el proceso de evaluación y permite dotar al alumnado de un mayor grado de libertad a la hora de plantear los trabajos, no acotando demasiado el camino a seguir, aunque sí fijando una línea de actuación que permite alcanzar la máxima puntuación final.

Para la evaluación de la propuesta y conocimiento por parte del profesorado de las impresiones percibidas por el estudiantado tras la realización del trabajo con metodología ApS, se ha diseñado un cuestionario que permite conocer de primera mano las cuestiones más relevantes sobre el desarrollo, organización, percepción sobre los temas propuestos, acciones sociales realizadas y alcance de la actuación, entre otras cuestiones, y que sirvan como punto de partida para mejorar la experiencia en futuros cursos académicos. En síntesis, el contenido del cuestionario de autoevaluación se puede esquematizar a través de los siguientes ítems como se muestra en la Figura 3.

ÍTEMS para la construcción del cuestionario de autoevaluación
Interés inicial e impresiones hacia la asignatura.

$>$ Interés final e impresiones sobre la asignatura.

$>$ Grado de satisfacción general con la impartición de clases de la asignatura.

Conocimiento previo de la metodología ApS.

$>$ Percepción acerca del nivel de retención del conocimiento/aprendizaje mediante el ApS.

> Grado de satisfacción general con metodología ApS en la asignatura.

$>$ Percepción de utilidad del servicio prestado a la comunidad/colectivo.

Valoración del funcionamiento del trabajo en grupo.

$>$ Ideas y intereses para futuros trabajos con metodología ApS.

Fig. 3. Esquema que recoge los ÍTEMS más importantes para la elaboración del cuestionario de autoevaluación del alumnado.

Por otro lado, además de servir como fuente de ideas para la mejora de la propuesta donde los estudiantes se expresen libremente. Un cuestionario bien planteado en términos como los que recoge la Figura 3, es de gran utilidad al profesorado para conocer el nivel de compromiso adquirido por el estudiantado con la actividad, así como, el nivel de contacto con la realidad de su entorno que han tenido durante la realización del proyecto, ya que no todos los grupos tienen porqué haberse involucrado por igual en la propuesta desde el inicio.

A modo de ejemplo, en la Figura 4 se muestra una propuesta de aplicación realizada por el alumnado de la asigantura de Gestión y Control de la Calidad. En dicha propuesta se aúnan los objetivos previstos para la asignatura, como es el conocimiento y manejo de las siete herramientas clásicas de la calidad (en este caso, un Diagrama de Ishikawa), con el objetivo medioambiental de conocer la eficiencia en la colocación y gestión de las papeleras en el Campus de Montegancedo. Se trata de un claro ejemplo que de actuación en línea con los ODS 13 y 15 (Acción por el clima y Vida de ecosistemas terrestres), que sirve de punto de partida para la elaboración de un cuestionario posterior que permita la recogida de datos y la recolocación de aquellos puntos de recepción de residuos que se encuentren fuera del alcance habitual del estudiantado. Con este tipo de acciones se presta un servicio útil para la Comunidad Universitaria, ya que el alumnado es el principal usuario de las instalaciones del Campús y son nuestros y nuestras estudiantes quienes mejor conocen las limitaciones del mismo. No obstante, se han realizado otras propuestas como: Desarrollo de aplicaciones para compartir coche y reducir emisiones, mejora del reciclaje de los productos en la cafetería, transición energética de los eficicios hacia un modelo energético autosostenible...etc. 


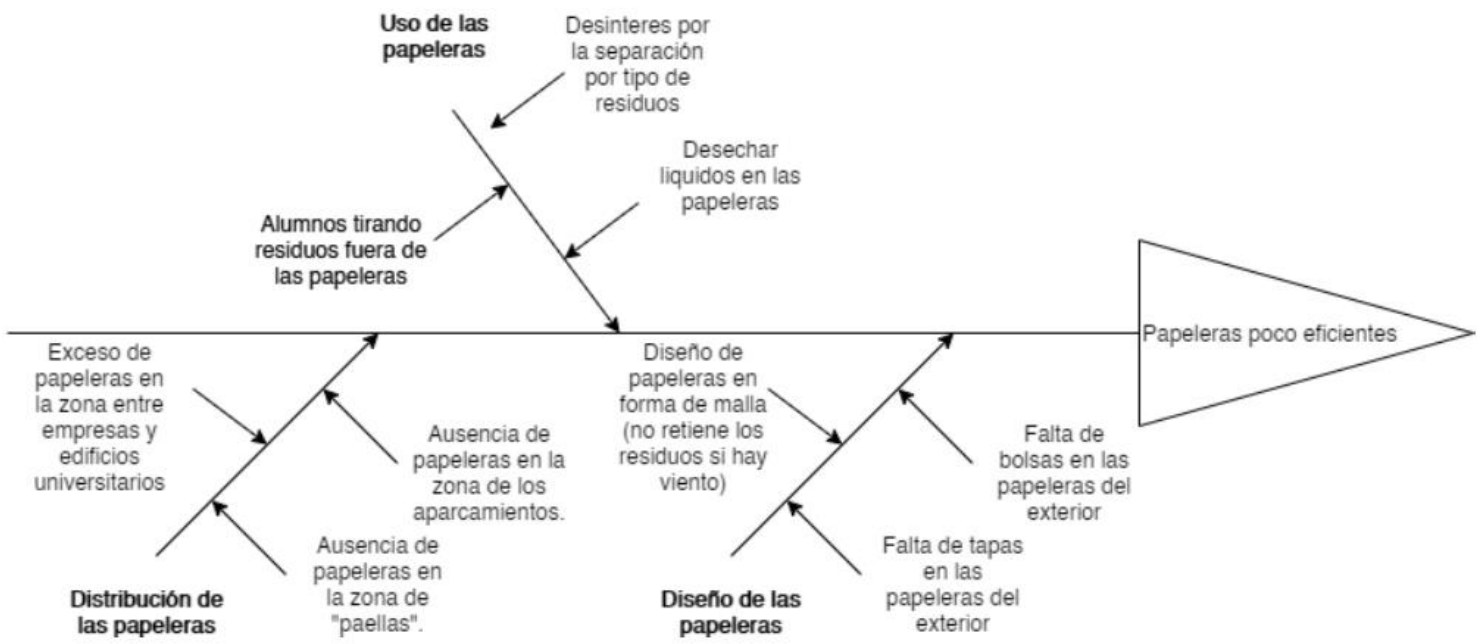

Fig. 4. Diagrama en espina de pez o de Ishikawa realizado por los y las estudiantes de la E.T.S. de Ingenieros Informáticos para determinar las causas de la falata de eficiencia en las papeleras universitarias.

Finalmente, en la Figura 5, se muestran algunos de los resultados derivados de la encuesta de satisfacción que hasta la fecha se ha podido realizar al estudiantado.

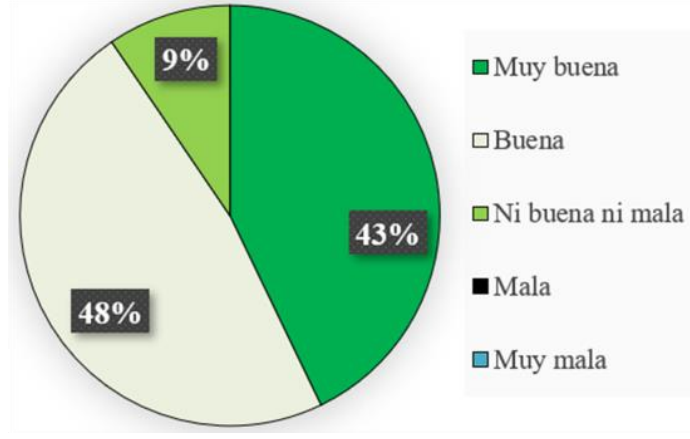

(a)

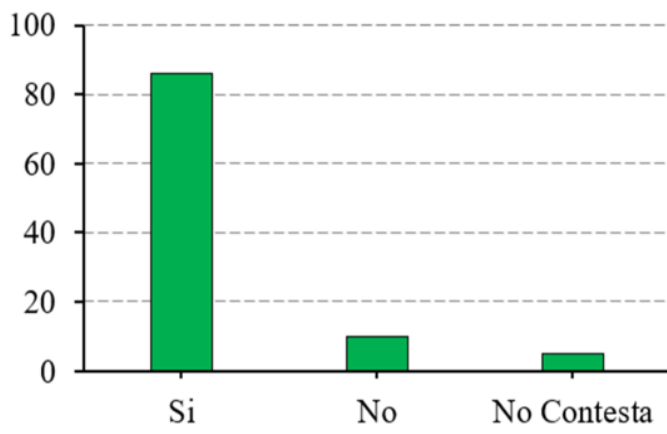

(b)

Fig. 5. Respuesta a la encuesta de satisfacción. (a) ¿Cómo valoras tu experiencia trabajando en grupo?; (b) ¿Recomiendas la repetición de esta metodología y este trabajo para cursos posteriores de esta asignatura?

Como se puede apreciar, en la Figura 5(a) se puede ver que el 91\% del estudiantado encuestado ha valorado como buena o muy buena su experiencia trabajando en grupo mediante la metodología ApS, no habiendo respuestas negativas a la pregunta. Este resultado es de gran utilidad para seguir trabajando en un proceso de mejora continua para aumentar la satisfacción de todo el estudiantado y la motivación e involucración con la propuesta que realizan. Asimismo, en la Figura 5(b) se aprecia como la gran mayoría del alumnado (más del 80\%), recomendaría la aplicación de la metodología ApS en cursos venideros. Este resultado muestra el éxito académico que se obtiene mediante la aplicación de los contenidos teóricos a la prestación de servicios prácticos, que sirven para cubrir alguna o varias necesidades sociales y que fomentan el desarrollo personal de los y las estudiantes.

\section{Conclusiones}

A lo largo de este trabajo el ApS se ha presentado como una metodología educativa con una finalidad social, que une dos elementos clave para el desarrollo de la persona: el compromiso social y el éxito académico.

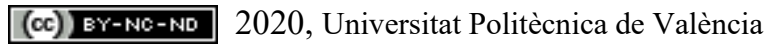


En este sentido, son muchos los centros y docentes que trabajan sin hacerlo extensivo el AprendizajeServicio, si bien es cierto, que este trabajo ha ayudado a acotar las pautas para definir la manera de llevar a cabo un proyecto docente en una asignatura de grado en ingeniería, sin riesgo de quedarse corto y ofrecer un mero voluntariado, o de excedernos por el contrario e involucrar a los estudiantes en un proyecto de investigación.

De manera eficaz se ha propuesto desde la asignatura de Gestión y control de la calidad una manera de realizar un proyecto integrado dentro de un semestre, que favoreciendo la participación directa en el alcance de alguno de los Objetivos para el Desarrollo Sostenible de las Naciones Unidas, se realice de forma cercana a través de una actuación directa en el Campus de la Universidad. Se ha mostrado, como se puede dotar al alumnado de cierto empoderamiento en el ámbito universitario, siendo los estudiantes quienes elijan la acción social a realizar y siendo conocedores del método de evaluación a seguir mediante la facilitación de una rúbrica de evaluación. Esta acción de empoderamiento por parte del estudiantado ha aumentado su grado de satisfacción con la metodología ApS, habiendo trabajado en grupo comodamente y obteniendo buenos resultados, así como, recomendando la aplicación de la propuesta en cursos venideros como muestran los resultados derivados de la encuesta de satisfacción realizada hasta la fecha.

Este tipo de propuestas docentes, deben verse como una oportunidad educativa en la cual el alumnado se puede comprometer realizando una mejora en su población o entorno más cercano, incluso abordando conceptos tan genéricos como los ODS. Se trata de una práctica académica que permite ajustar su duración en base al alcance del proyecto de manera sencilla, y dotar de un aprendizaje experiencial al alumnado incluso en asignaturas eminentemente teóricas como la que en este trabajo se ha planteado. No hemos de olvidar que el fin de la educación es formar ciudadanas y ciudadanos competentes.

\section{Referencias}

DE SANTIAGO NOCITO, A. M. et al. (2019). "El aprendizaje por servicio: un modelo para la incorporación de competencias clínicas en medicina". RIECS, Revista de Investigación y Educación en Ciencias de la Salud, vol. 4, issue 1, p. 35-39.

DEL RÍO, R. (2016). "Aplicación de la metodología aprendizaje servicio en un curso de Química. RIDAS, Revista Iberoamericana de Aprendizaje Servicio, vol. 2, p. 172-179.

FERNÁNDEZ, R. (2008). Principios y técnicas de calidad y su gestión en edificación. Madrid: Fundación General UPM.

LÓPEZ-DÓRIGA, M. y MARTÍN, X. (2018). "El aprendizaje servicio como práctica inclusiva. RIDAS, Revista Iberoamericana de Aprendizaje Servicio, vol. 6, p. 154-169.

MAROTO, S. (2018). "La educación se mueve en el territorio. Estrategias locales de aprendizaje-servicio". RIDAS, Revista Iberoamericana de Aprendizaje Servicio, vol. 6, p. 19-32.

MEDINA, R. (2012). "Service-Learning as inclusive strategy to Overcoming Barriers to learning and participation". Revista de Educación Inclusiva, vol. 5, issue 1, p. 71-82.

MIRANDA, F., CHAMORRO, A. y RUBIO, S. (2017). Calidad y Excelencia. Madrid: Delta Publicaciones.

PUIG, J. M. (coord.) (2009). Aprendizaje-Servicio (ApS), Educación y compromiso civico. Barcelona: Graó.

PUIG, J. M. (2018). "Difusión y arraigo del aprendizaje-servicio". Revista Iberoamericana de Aprendizaje Servicio, vol. 6, p. 88-102.

URUÑUELA, P. M. (2018). La Metodología del Aprendizaje-Servicio. Madrid: Narcea. 